\title{
Assessment of SLW model in 3D non-gray gas radiation calculation
}

\author{
Masoud Darbandi' ${ }^{\text {, Mohamad Bagher Barezban', Gerry E. Schneider }}{ }^{2}$ \\ ${ }^{1}$ Department of Aerospace Engineering, Centre of Excellence in Aerospace Systems, Sharif University of Technology \\ Azadi Ave., P. O. Box 11365-11155, Tehran, Iran \\ darbandi@sharif.edu; barezban@gmail.com \\ ${ }^{2}$ Department of Mechanical and Mechatronic Engineering, University of Waterloo \\ Waterloo, Ontario, N2L 3G1, Canada \\ gerry.schneider@uwaterloo.ca
}

\begin{abstract}
In this paper, the radiation heat transfer is calculated numerically in a three dimensional enclosure containing non-gray gases. The radiation heat transfer equation is solved using the finite-volume formulation of discrete ordinate method and the SLW model is employed to calculate the radiation absorption coefficients. Three cases are simulated here including the isothermal/homogeneous, isothermal/nonhomogeneous, and the non-isothermal/homogeneous media. The calculated radiation source terms and the wall heat fluxes are compared with the results of SNB method. It is shown that the SLW results are in very good agreement with the SNB in case of isothermal/homogeneous. In the two other cases, the SLW results show more deviations than this one. It is because the SLW formulation employs more additional assumptions to calculate the radiation in media with non-uniform distribution of temperature or mixture composition and multicomponent gas mixture. However, comparing the results of SLW with those of the WSGG model, it indicates that the former case would be more accurate. This is because the parameters of SLW model are calculated directly from the high resolution spectroscopic database of gas molecules.
\end{abstract}

Keywords: Radiation heat transfer, Radiation absorption coefficient, Spectral radiation, SLW model.

\section{Introduction}

In the global models, the RTE is integrated over the absorption coefficient instead of the wavenumber. The most popular global model called Weighted-Sum-of-Gray-Gases model (WSGGM) was first proposed within the framework of the zonal method [1], but it was demonstrated that it can be applied with general RTE solution method [2]. It represents the spectrum with a few gray gases, ordinary 4 or 5, that occupy certain portions of the spectrum. WSGGM assumes that total emissivity of gas mixture can be represented by the sum of a gray gas emissivity weighted with a temperature dependent factor. The gray gas emissivity is expressed in terms of a temperature-independent absorption coefficient and the weighting factors are given by polynomials in gas temperature with associated polynomial coefficients [3]. The gray gases absorption coefficients and the weighting factors polynomial coefficients are obtained using curve fitting of the model to total emissivity obtained from experimental measurements, spectroscopic databases or narrow and wide band models.

References [4-6] have already introduced different approaches to generate the WSGG models directly from the lineby-line spectra of participating gas mixture components. This Spectral Line-Based WSGG model is called SLW. In the SLW formulations, the absorption coefficients of gray gases are chosen and the corresponding weighting factors are calculated from the detailed spectroscopic data of gas molecules. The applicability of SLW in non-isothermal and nonhomogeneous media is possible using the reference approach $[7,8]$. The multiplication approach is used in development of the SLW model to allow for mixtures of participating gases [9, 10] and mixtures of gases including soot [11].

In this study, radiation heat transfer equation is solved in a $3 \mathrm{D}$ enclosure containing non-gray gas mixture. It aims to analyze the accuracy of SLW model implemented in the finite-volume formulation of discrete ordinate method for the RTE solution. The main purpose of the present study is to assess the accuracy of SLW model in 3D non-gray gas radiation calculation.

\section{SLW Formulation}

The radiative heat transfer equation in a non-scattering medium can be simplified to [13] 


$$
\frac{d I_{\eta}}{d s}=\kappa_{\eta}\left(I_{b \eta}-I_{\eta}\right)
$$

where $I_{\eta}$ is the spectral radiation intensity at the wavenumber $\eta$ and along the path length $\mathrm{s}, I_{b \eta}$ is the Plank blackbody intensity and $\kappa_{\eta}$ is the spectral absorption coefficient. It has been shown that the total spectrum can be divided into J gray gases and one RTE is solved for each [2] as follows:

$$
\frac{d I_{j}}{d s}=\kappa_{j}\left(a_{j} I_{b}-I_{j}\right), \quad j=0,1, \ldots, J
$$

where $I_{j}, \kappa_{j}$ and $a_{j}$ are respectively the intensity, absorption coefficient and corresponding blackbody weighting factor for the $\mathrm{j}$-th gray gas and $I_{b}$ is the total intensity of blackbody radiation given by $\sigma T^{4} / \pi$. The total radiation intensity $I$ is obtained by the summation of the solutions of Eq. (2) over all gray gases using

$$
I=\sum_{j=1}^{J} I_{j}
$$

The gas absorption cross-section spectrum $C_{\eta}$ obtained from high resolution HITEMP 2010 spectroscopic database [14] by Lorentz profile [15]. For calculation of $\kappa_{j}$ and $a_{j}$ parameters, $C_{\eta}$ is divided into J intervals using the supplemental absorption cross-sections $\tilde{C}_{j}$ given by Eq. (4). The supplemental absorption cross-sections have been equally and logarithmically spaced between the minimum $\tilde{C}_{0}=C_{\min }$ and the maximum $\tilde{C}_{J}=C_{\max }$ values, which effectively represent the entire absorption spectrum $C_{\eta}$.

$$
\tilde{C}_{j}=C_{\min }\left(C_{\max } / C_{\min }\right)^{j / J}, \quad j=0,1, \ldots, J
$$

The logarithmic mean of the adjacent supplemental absorption cross-sections represents the absorption coefficient of gray gas $\mathrm{j}$ as follows:

$$
\kappa_{j}=N Y \sqrt{\tilde{C}_{j-1} \tilde{C}_{j}}
$$

where $Y$ is the gas mole fraction in the mixture and $N$ is its molar density defined by $p N_{A} / R_{u} T$, where $N_{A}$ is Avogadro's number, $R_{u}$ is the universal gas constant, $\mathrm{p}$ is the gas mixture pressure, and $\mathrm{T}$ is its temperature.

The weighting factors $a_{\mathrm{j}}$ are determined as a difference between values of the absorption-line blackbody distribution function (ALBDF) $F\left(C, T_{g}, T_{b}, Y\right)$ at the adjacent supplemental absorption cross-sections using

$$
a_{j}=F\left(\tilde{C}_{j}, T_{g}, T_{b}, Y\right)-F\left(\tilde{C}_{j-1}, T_{g}, T_{b}, Y\right)
$$

and the ALBDF is defined as:

$$
F\left(C, T_{g}, T_{b}, Y\right)=\frac{1}{I_{b}\left(T_{b}\right)} \int_{\left\{\eta: C_{\eta}\left(T_{g}, Y\right) \leq C\right\}} I_{b \eta}\left(T_{b}, \eta\right) d \eta
$$


The multiplication approach $[9,10]$ can be used to obtain the ALBDF for mixture of participating gases. In this approach, the ALBDF for gas mixture (for example the mixture of water vapor $\mathrm{H}_{2} \mathrm{O}$ and carbon dioxide $\mathrm{CO}_{2}$ ) is the multiplication of ALBDF of the individual gas mixture components, i.e,

$$
F_{\text {mix }}\left(\mathrm{C}, T_{g}, T_{b}, Y\right)=F_{w}\left(\frac{C}{Y_{w}}\right) F_{c}\left(\frac{C}{Y_{c}}\right)
$$

Here, we used the tabulated data generated by Pearson [16] to calculate the ALBDF of participating gases. For radiation calculations in nonisothermal and nonhomogeneous media, we used reference state approach [7, 8]. In this approach the reference temperature $T_{r e f}$ and the reference mole fraction of participating gases $\boldsymbol{Y}_{\text {ref }}$ is defined as the volume averaged of the corresponding local variables in the whole domain. Then, the local supplemental absorption cross-sections $\tilde{C}_{j}^{l o c}$ is obtained by implicitly solving of the following equation:

$$
F\left(\tilde{C}_{j}^{l o c}, T_{g}=T_{l o c}, T_{b}=T_{r e f}, \boldsymbol{Y}=\boldsymbol{Y}_{l o c}\right)=F\left(\tilde{C}_{j}^{r e f}, T_{g}=T_{\text {ref }}, T_{b}=T_{\text {ref }}, \boldsymbol{Y}=\boldsymbol{Y}_{\text {ref }}\right)
$$

The local absorption coefficients $\kappa_{j}^{l o c}$ and weights ${a_{j}}^{l o c}$ are calculated from

$$
\begin{gathered}
\kappa_{j}^{l o c}=N\left(T^{l o c}\right) Y^{l o c} \sqrt{\tilde{C}_{j-1}^{l o c} \tilde{C}_{j}^{l o c}} \\
a_{j}^{l o c}=F\left(\tilde{C}_{j}^{r e f}, T_{g}=T_{r e f}, T_{b}=T_{l o c}, \boldsymbol{Y}=\boldsymbol{Y}_{r e f}\right)-F\left(\tilde{C}_{j-1}{ }^{r e f}, T_{g}=T_{r e f}, T_{b}=T_{l o c}, \boldsymbol{Y}=\boldsymbol{Y}_{r e f}\right)
\end{gathered}
$$

In this study, the RTE is solved here using the finite-volume formulation of the discrete ordinate (DO) method [17].

\section{Description of Test Problem}

The test problem is a $2 \mathrm{~m} \times 2 \mathrm{~m} \times 4 \mathrm{~m}$ rectangular enclosure in the $\mathrm{x}, \mathrm{y}$ and $\mathrm{z}$ directions, respectively, where the black and cold walls are at $300 \mathrm{~K}$. Three cases are considered here. They are described in Table 1. For all the cases, the pressure of the gas mixture in the enclosure is 1 atm. Liu [12] calculated narrow-band averaged radiation heat transfer equation in this 3D enclosure using ray-tracing method along with SNB model for nongray gas radiation. The reported radiation source terms and the wall heat fluxes can be used as a benchmark for less accurate global models.

Table 1: Conditions of three test cases, [12].

\begin{tabular}{|cll|}
\hline Case & Temperature distribution & Gas mixture compositions \\
\hline 1 & isothermal, $1000 \mathrm{~K}$ & Homogeneous, pure $\mathrm{H}_{2} \mathrm{O}$ \\
2 & isothermal, $1000 \mathrm{~K}$ & Nonhomogeneous, $\mathrm{H}_{2} \mathrm{O} / \mathrm{N}_{2}$ mixture \\
3 & Non-isothermal & Homogeneous, $\mathrm{CO}_{2} / \mathrm{H}_{2} \mathrm{O} / \mathrm{N}_{2}$ mixture \\
\hline
\end{tabular}

In test case 2 , the mole fraction of $\mathrm{H} 2 \mathrm{O}$ varies in $\mathrm{z}$ direction with $\mathrm{xH} 2 \mathrm{O}=4(\mathrm{z} / \mathrm{Lz})(1-\mathrm{z} / \mathrm{Lz})$ relation, where $\mathrm{Lz}=4 \mathrm{~m}$. In the test case 3 , the medium is a homogeneous mixture of $0.1 \mathrm{CO} 2+0.2 \mathrm{H} 2 \mathrm{O}+0.7 \mathrm{~N} 2$ (mole basis). The gas temperature is non-uniform but distributed symmetrically about the centerline of the enclosure, centerline in $\mathrm{z}$ direction, with $\mathrm{T}=(\mathrm{Tc}-$ $\mathrm{Te}) \mathrm{f}(\mathrm{r} / \mathrm{R})+\mathrm{Te}$, where $\mathrm{Tc}$ is the gas temperature along the enclosure centerline and Te is the exit temperature at $\mathrm{z}=4 \mathrm{~m}$. Inside the circular region of the cross section of the enclosure, the gas temperature variations is defined by $f(r / R)=1-$ $3(r / R) 2+2(r / R) 3$, where $r$ is the distance from the centerline and $R$ is the radius of circular region $(R=1 \mathrm{~m})$. The gas temperature outside the circular region is uniform at the value of the exit temperature. The centerline temperature increases linearly from $400 \mathrm{~K}$ at the inlet $(\mathrm{z}=0)$ to $1800 \mathrm{~K}$ at $\mathrm{z}=0.375$, then decreases linearly to $800 \mathrm{~K}$ at the exit. 


\section{Results and Discussion}

The numerical results are obtained using $50 \times 50 \times 50$ uniform spatial grids. The RTE is solved by the finite volume based discrete ordinate method, DO, with T4 approximation for angular discretization which consists of 128 directions. The SLW with 20 gray gases are used to solve the RTE in 3D rectangular enclosure.

Fig. 1 shows the calculated radiative source term along the enclosure centerline, $(1 \mathrm{~m}, 1 \mathrm{~m}, \mathrm{z})$, and the wall heat flux along $(2 \mathrm{~m}, 1 \mathrm{~m}, \mathrm{z})$ for the first case. As can be seen, the present SLW results show very good agreement with the SNB results of Liu [12] in case of isothermal/homogeneous pure $\mathrm{H}_{2} \mathrm{O}$.

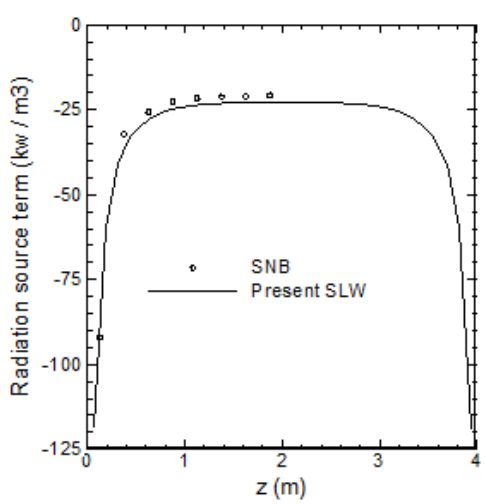

a)

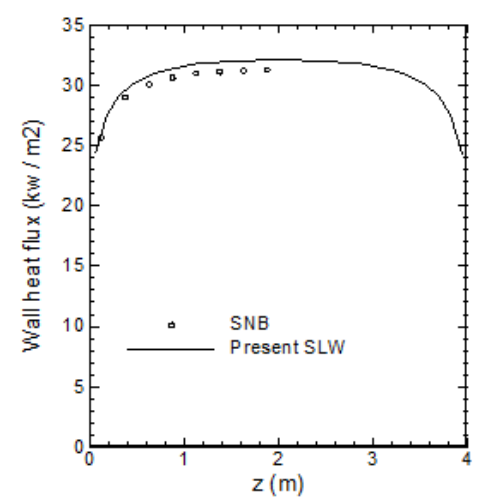

b)

Fig. 1: Distribution of a) radiation source term along the centerline and b) wall heat flux along $(2 \mathrm{~m}, 1 \mathrm{~m}, \mathrm{z})$ for test case 1 .

Fig. 2 shows the calculated radiative source term along the centerline $(1 \mathrm{~m}, 1 \mathrm{~m}, \mathrm{z})$ and wall heat flux along $(2 \mathrm{~m}$, $1 \mathrm{~m}, \mathrm{z})$ for the case 2. In this case, the temperature is uniform but the mole fraction ${ }^{2} \mathrm{H}_{2} \mathrm{O}$ varies in $\mathrm{z}$ direction. Reference approach is used here for spatial variation of $\mathrm{H}_{2} \mathrm{O}$ mole fraction. Along the centerline and at $\mathrm{z}=2 \mathrm{~m}$, the differences between the SNB and the SLW results are maximum and the SLW absolutely overpredicts the radiation source term and the wall heat flux about $10 \%$ and $8.5 \%$, respectively. The over-prediction of SLW in case 2 is mainly caused by the ideal spectrum assumption used in the reference approach.

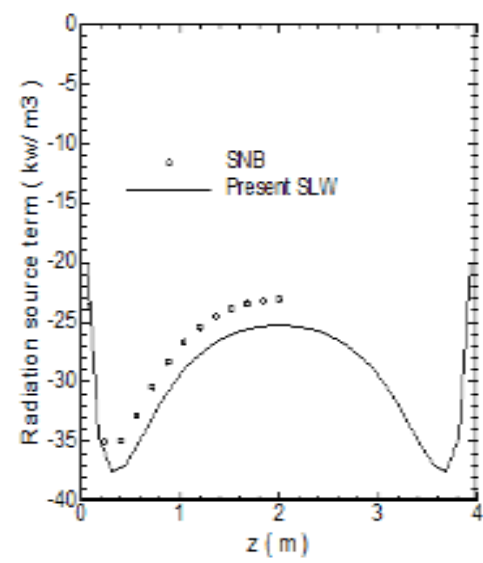

a)

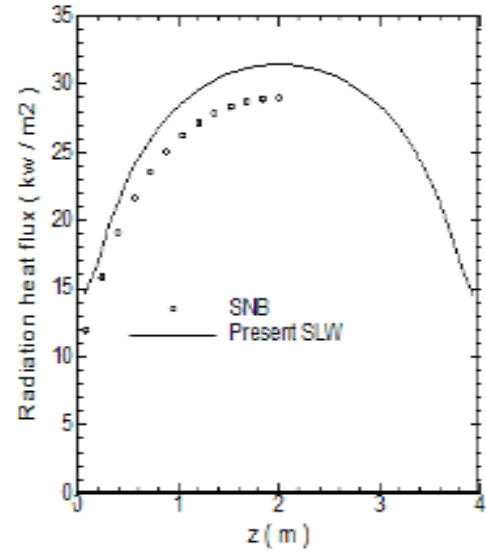

b)

Fig. 2: Distribution of a) radiation source term along the centerline and b) wall heat flux along ( $2 \mathrm{~m}, 1 \mathrm{~m}, \mathrm{z})$ for test 2 . 
For the third test case, the calculated radiative source terms along centerline $(1 \mathrm{~m}, 1 \mathrm{~m}, \mathrm{z})$ are shown in Fig. 3. In addition to SLW, the results of the radiation heat transfer calculations with WSGG model are shown in this figure. In this case, the participating media is homogeneous but nonisothermal. Reference and multiplication approaches are used for spatial variation of temperature and multicomponent participating gas mixture, respectively. The SLW results at the maximum value (at $\mathrm{z}=0.5 \mathrm{~m}$ ) would overpredict the SNB results about $20 \%$. In the reference approach, we used the ideal spectrum assumption, where the multiplication approach was based on the assumption that the spectra of different species included in the gas mixture are statistically independent. These two approximations increased the errors in the SLW predictions. However, in comparison with the WSGG, the SLW predictions are in more agreement with the SNB results, as can be concluded from Fig. 3. This is because the model parameters of SLW are calculated directly from the highresolution spectroscopic database of the gas molecules.

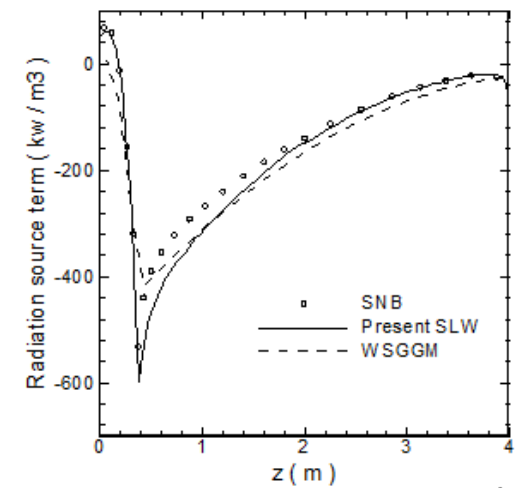

Fig. 3: Distribution of radiation source terms along the centerline for test case 3.

\section{Conclusion}

The radiation heat transfer was numerically calculated in a 3-D enclosure containing various homogenous/nonhomogeneous and isothermal/nonisothermal participating gas mixtures. The SLW model was employed in DO method to solve the RTE in non-gray media. The results of present radiation calculations were compared with the more accurate SNB calculations. The calculations showed that the SLW predictions were in very good agreement with those of the SNB in case of predicting the isothermal/homogeneous pure gas media. In cases with the spatial variations in temperature or mixture composition, the ideal spectrum assumption in the reference approach caused deviations of the results. On the other hand, employing the multiplication approach with the assumption of statistically independency of the spectra of different species, it increased the errors of SLW results more and more. However, this study indicated that the SLW would predict the radiation source terms more accurate than the WSGG model in non-gray media modeling. This is because the parameters of SLW model were calculated directly from the high resolution spectroscopic database of gas molecules. In addition, this research showed that the SLW model overpredicts the absolute radiation source terms and the wall heat fluxes (which must be considered in radiation calculations) in all cases.

\section{Acknowledgements}

The authors from Sharif University of Technology would like to thank the financial support received from the Deputy of Research and Technology in Sharif University of Technology. It is greatly acknowledged.

\section{Nomenclature}

$T=$ temperature

$Y \quad=$ species mole fraction

$\mathrm{a} \quad=$ gray gas weighting factor

$F \quad=$ absorption line blackbody distribution function 


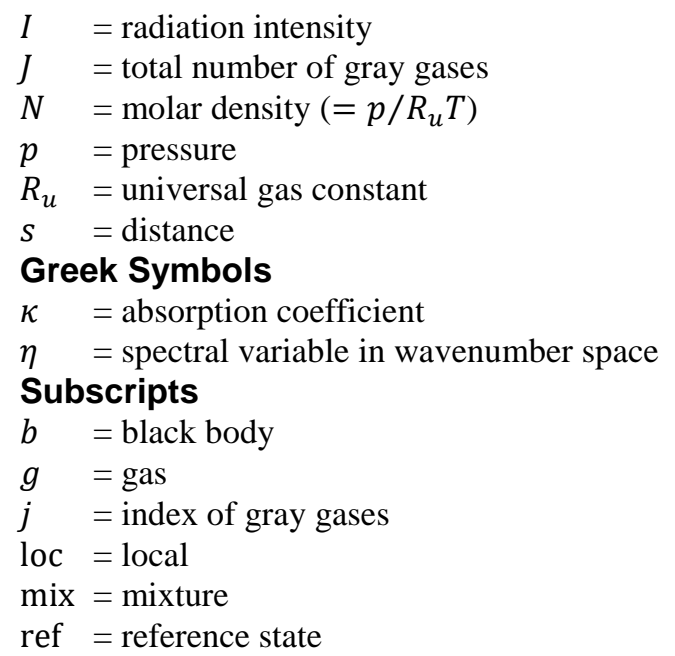

\section{References}

[1] H. C. Hottel and A. F. Sarofim, Radiative Transfer. New York: McGraw- Hill, 1967.

[2] M. F. Modest, "The Weighted-Sum-of-Gray-Gases Model for Arbitrary Solution Methods in Radiative Transfer," Journal of Heat Transfer, vol. 113, no. 3, pp. 650-656, 1991.

[3] T. F. Smith, Z. F Shen and J. N. Friedman, "Evaluation of Coefficients for the Weighted Sum of Gray Gases Model," Journal of Heat Transfer, vol. 104, pp. 602-608, 1982.

[4] M. K. Denison and B. W. Webb, "A spectral line-based weighted-sum-of-gray-gases model for arbitrary RTE solvers," Journal of Heat Transfer, vol. 115, pp. 1004-1012, 1993.

[5] M. K. Denison and B. W. Webb, "An absorption-line blackbody distribution function for efficient calculation of total gas radiative transfer," J. Quant. Spectrosc. Radiat. Transfer, vol. 50, pp. 499-510, 1993.

[6] M. K. Denison and B.W. Webb, "Development and Application of an Absorption-Line Blackbody Distribution Function for $\mathrm{CO}_{2}$," Int. J. Heat Mass Transfer, vol. 38, pp. 1813-1821, 1995.

[7] M. K. Denison, "A spectral line-based weighted-sum-of-gray-gases model for arbitrary RTE solvers," Ph.D dissertation, Department of Mechanical Engineering, Brigham Young University, Provo, UT, USA.

[8] M. K. Denison and B.W. Webb, "The spectral line based weighted-sum-of- gray-gases model in non-isothermal nonhomogeneous media," Journal of Heat Transfer, vol. 117, pp. 359-365, 1995.

[9] M. K. Denison and B. W. Webb, "The Spectral-Line Weighted-Sum-of-Gray-Gases Model for $\mathrm{H}_{2} \mathrm{O} / \mathrm{CO}_{2}$ Mixtures," Journal of Heat Transfer, vol. 117, pp. 788-792, 1995.

[10] V. P. Solovjov and B. W. Webb, "SLW modeling of radiative transfer in multicomponent gas mixtures," J. Quant. Spectrosc. Radiat. Transfer, vol. 65, pp. 655-672, 2000.

[11] V. P. Solovjov and B. W. Webb, "An Efficient Method for Modeling of Radiative Transfer in Multicomponent Gas Mixtures with Soot Particles," Journal of Heat Transfer, vol. 123, pp. 450-457, 2001.

[12] F. Liu, "Numerical Solutions of Three-Dimensional Non-Grey Gas Radiative Transfer Using the Statistical NarrowBand Model," Journal of Heat Transfer, vol. 121, pp. 200-203, 1999.

[13] M. F. Modest, Radiative Heat Transfer. New York: Academic, 2013.

[14] L.S. Rothman, I. E. Gordon, R. J. Barber, H. Doth, R. R. Gamache, A. Goldman, V. I. Perevalov, S. A. Tashkun, J. Tennyson, "HITEMP, the high-temperature molecular spectroscopic database," J. Quant. Spectrosc. Radiat. Transfer, vol. 111, no. 15, pp. 2139-2150, 2010.

[15] L. J. Dorigon, G. Duciak, R. Brittes, F. Cassol, M. Galarça, F. H. R. França, "WSGG correlations based on HITEMP2010 for computation of thermal radiation in non-isothermal, non-homogeneous $\mathrm{H} 2 \mathrm{O} / \mathrm{CO} 2$ mixtures," Int. J. Heat Mass Transfer, vol. 64, pp. 863-873, 2013. 
[16] J. T. Pearson, B. W. Webb, V. P. Solovjov and J. Ma, "Efficient representation of the absorption line blackbody distribution function for $\mathrm{H}_{2} \mathrm{O}, \mathrm{CO}_{2}$, and $\mathrm{CO}$ at variable temperature, mole fraction, and total pressure," J. Quant. Spectrosc. Radiat. Transfer, vol. 138, pp. 82-96, 2014.

[17] E. H. Chui, G. D. Raithby, "Computation of Radiant Heat Transfer on a Non-Orthogonal Mesh Using the FiniteVolume Method," Numerical Heat Transfer, Part B, vol. 23, pp. 269-288, 1993. 